\title{
Accretion Disks and Magnetic Fields
}

\author{
a Europhysics Study Conference
}

\section{Gaetano Belvedere, Catania}

(Istituto di Astronomia)

The Europhysics Study Conference "Accretion Disks and Magnetic Fields in Astrophysics" was held in Noto, Sicily, Italy from 16 to 21 June 1988 at the Centro Studi Superiori e Ricerche, a young and promising scientific institution under the patronage of the Noto City Council. The Conference was attended by about fifty participants from Canada, China, Denmark, France, FRG, Italy, Japan, the Netherlands, Spain, Switzerland, U.K. and U.S.A. The Scientific Organizing Committee was composed of G. Belvedere (Chairman), A.O. Benz, R.N. Henriksen, R.V. Lovelace, F. Pacini, S.N. Shore, H.C. Spruit, W.M. Tscharnuter and N.O. Weiss, while the Local Organizing Committee included A.M. Anile, M. Rodono' (Director of the Catania Astrophysical Observatory) and O. Muscato.

The purpose of the Conference was to try to form some unified picture of the dynamical interaction, under many different astrophysical conditions, of rotation, turbulence and magnetism. To achieve this, we brought together colleagues working on different aspects of different objects, ranging from star formation, solar and stellar activity, close binaries, to neutron stars, $\mathrm{X}$-ray galactic sources and actives galaxies.

Magnetic dynamo processes similar to those used to explain solar and stellar activity, have been invoked recently in studies of galactic magnetic fields, dynamical processes occurring in the atmospheres of neutron stars, and the origin and maintenance of jets in protostellar objects and active galactic nuclei (AGN). The primary role played by the magnetic field is amply shown by the relativistic and non-thermal processes observed in solar and stellar flares, in cataclysmic variables, in neutron star magnetospheres and in the powerful central engines of active galaxies, where a supermassive black hole is supposed to exist. Moreover, instabilities in solar energetic phenomena and protostellar clouds, as well as in the formation of disks by accretion of matter round compact objects as seen in the binary galactic X-ray sources, have shed light on some of the properties of much more massive disks existing around huge collapsed objects in the very core of qua- sars, lacertids, strong emitting radiogalaxies and Seyferts. The choice of topics aimed to emphasize the universality of the underlying physical processes and mechanisms, despite different masses, sizes, energy contents and outputs, and characteristic timescales.

The Conference was structured into three principal sections: solar and stellar activity; protostellar and binary system disks; AGN disks. In the context of the first, particular attention was given to the physics of convection and magnetism in rotating stellar shells, to magnetohydrodynamical (MHD) turbulence and dynamo processes in stars, with reference to magnetic activity including stellar flares. The talks given in the second section dealt with basic accretion disk theory for binary systems with collapsed companions, dwarf nova and cataclysmic variable disks; the role of magnetic fields in determining disk structure and wind, turbulent generation of high velocity flows and jets in clouds, disks and magnetospheres, MHD processes in disks and jets, with particular reference to close binaries and protostars. The last section included such topics as the morphology and physics of AGN and related jets, the central powerhouse of $A G N$, the disk and jet $\mathrm{MHD}$ and the relativistic processes occurring in the rotating magnetosphere of the inner core of active galaxies.

Particular interest was expressed by the participants in two topical joint discussions on the subjects of Stellar Disks and AGN Disks, during which the main points raised were:

- The necessity for a more sophisticated modelling of accretion disks, leading to the simultaneous solution of all relevant equations, including eventually the $\mathrm{MHD}$ if the role of the large scale magnetic fields appears to be significant in the physical description (accretion disk dynamo).

- The keplerian rotation does not represent the most general angular velocity distribution inside the disk, especially in the case of thick disks.

- Thin disk models may suffice for certain binary systems, but, in the case of AGN the peculiar physical scenario requires thick disk (accretion torus) mo- delling. In this regard it has been pointed out that plasma radiative effects in the AGN disk envelope are determinant.

- The interaction between the disk and the magnetic field of the compact object (white dwarf or neutron star) appears to be relevant, and in some circumstances, disk formation may be inhibited by the collapsed companion's strong magnetic field. Moreover, if disks do have a large scale dynamo regulated magnetic field, the interaction between the latter and the collapsed companion's magnetic field should be investigated.

- As to the scaling between disk models for galactic and extragalactic sources, some difficulties have been raised, based on the strong dependence of the physical description on the peculiar environmental scenario, especially the source and rate of accretion, and the disk temperature, density and opacity. They should not scale simply with the mass of the central object (neutron star or supermassive black hole). However, scaling is allowed for systems of comparable size and mass and, in general, the magnetic field should scale as the size of the central object.

- Acceleration and collimation for both protostar and AGN jets depend strongly on the accretion rate, the jet density and opacity, and the physical properties of the environment. The magnetic field seems not to be dynamically relevant to the jet motion, and there is no compelling observational evidence for magnetic collimation in AGN jets. Thus, mechanisms for magnetic field dissipation should be included when carrying out numerical simulations of MHD jets.

- Finally, the existing observations in favour of or against spinar (clean) models (low entropy engines) and gravar (dirty) models (high entropy engines) have been carefully examined. In the former case, the primary AGN energy source is rotation, in the latter, it is conversion of gravitational binding energy. However, the argument has not been settled, as no strong evidence has been found against either model.

The Conference Proceedings, which are being edited by the author will be published by Kluwer (Reidel), in the Astrophysics and Space Science Library, in mid 1989. 